MATEC Web of Conferences 22,02013 (2015)

DOI: $10.1051 /$ matec conf/ 20152202013

(C) Owned by the authors, published by EDP Sciences, 2015

\title{
Threat Degree Analysis of the Multi-targets in Naval Gun
}

\author{
Hao Sun \& Yong Li \\ Department of Electronic Information, Northwestern Polytechnical University, Xi'an, Shaanxi, China
}

\begin{abstract}
For solving the problem of the multi-targets threat from the air, this paper analyzes the threat degree of many kinds of different moving stages in naval gun system. The authors analyze the drawbacks of previous methods and provide a new analytical model which is based on the theory of grey correlation and correlation coefficient. Using the new mathematical threat degree analysis model, the authors verify the new model by practical experiment and practical applications. In addition, this paper compares the merit and demerit of traditional method and the new method of analytical model. It turned out that the new model is better than previous ones and it can be used effectively and easily, and its consumption of system recourse is in theoretical range.
\end{abstract}

Keywords: system of naval gun; multi-targets; grey correlation; threat degree analysis

\section{INTRODUCTION}

With the increasing speed of military development, the way of war in modern society has become more and more various. For the air assault, enemies always use multi-headed missiles and multiple times of attack to destroy the targets. Hence, it has become an important factor for naval gun system analyzing and arranging the threat degree of air multi-targets ${ }^{[1][2][3]}$. Judgment in time will be an essential factor for warfare today. If the assessment of the threat degree had some distortion or error, there will be a disaster for our defense system. As for the naval gun system these days, more and more defense missions were arranged by the operational headquarters. For preventing the assault from enemy, and establishing a comprehensive defense system ${ }^{[4][5][6]}$, the naval force has to be the first defense system combating with enemy.

So how to give out the correct decision of threat degree has become the essential thing to be solved. In the past, we used several methods such as the previous experience, the short-cut route ${ }^{[7]}$, the Fuzzy Relation ${ }^{[8][9][10]}$, the method based on time and space ${ }^{[11][12]}$ and the Genetic Algorithm ${ }^{[13]}$ which cannot satisfy all the circumstances of modern warfare. Judgment which is based on previous experience always gives out the wrong result and will miss the right time to fire because its result included so much personal influence. In addition, the method which is based on short-cut route only takes one of many influencing factors of threat degree analysis into consideration. It only considers the short-cut route which only contains the influencing factors of slope distance and course angle. Moreover, the Fuzzy Relation, which uses a new fuzzy model to analyze the threat degree of every target, only considers the main influencing factor (the maximum influencing factor of the target). The result of the Fuzzy Relation analysis has some degrees of distortion due to its ignorance of other relevant factors.
Low relevant factors do not mean that they have no influence on the result, so we should not make the assessment only by the main one. In addition, the method based on time and space is combined with threat path, network and threat status to analyze the threat degree. Although this method takes network and threat status into consideration, it only uses the threat path as the threat degree assessment of practical target. The method of Genetic Algorithm also has limitations in practical applications. That is the limitation of their working circumstances and the consumption of system recourses.

Therefore, that how to analyze the threat degree of air multi-target effectively and acquire a timely reaction have become an essential thing for the research of naval gun defense system. This paper compares and analyzes previous algorithms, and uses a new analytical model of the grey correlation ${ }^{[14]}$ and correlation coefficient ${ }^{[15]}$ to analyze the threat degree. Grey correlation can give out the proper threat status of targets, and the correlation coefficient can provide the right relationship between influencing factors. The new model which uses relative functions to quantify all the factors can influence the judgment of threat degree in naval gun defense system. According to the quantification by the calculation of the new model, we can obtain the arrangement of the target threat sequence and simultaneously attack the multi-target by the defense system. As a result, through a lot of practical experiments and applications the new method has proved that it can be used to analyze all the factors, which ensure its accuracy and efficiency.

\section{THREAT DEGREE ANALYSIS}

\subsection{Influencing factors of threat degree of different moving stages}

We know that in the naval gun defense system, there 
are many kinds of different moving targets. Every target has its own moving stage which changes with time. Each factor of the target has its own threat degree. Defining and judging the threat degree timely is the essential mission for the defense system.

In each working period of defense system, the moving stage of each target is provided by tracking radar. Through the data provided by tracking radar, we can make the judgment of threat degree of each target. However, the tracking radar can only provides a limited number of information about the targets. Therefore, we must make use of every piece of useful information which is provided by it as much as possible.

In the naval gun system, factors which can influence the threat degree and the threat degree principle they obey are shown as follows:

(1) Slope distance: This factor is negatively correlated with threat degree. That is to say, the threat degree is increased with the decrease of slope distance, and vice versa.

(2) Flight speed: It is positively correlated with threat degree. That is, the threat degree is increased with the increasing flight speed.

(3) Flight altitude: The threat degree is increased if the distance between target and the defense system is decreased.

(4) Course angle: It represents the meaning of the angle between the flight direction and the point of the defense system. The target will be more offensive if the angle is becoming smaller. So, the threat degree is increased if the angle becomes smaller, and it will be more offensive to our defense system.

Factors provided by tracking radar are shown in Figure 1:

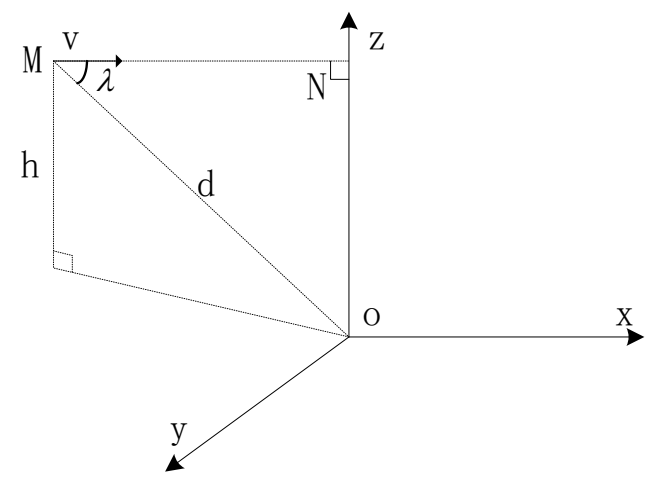

Figure 1. Factors in moving stages.

$O$ is the point of defense system, $M$ is the target point. So $d$ is the slope distance; $\mathrm{v}$ is the flight speed; $\mathrm{h}$ is the flight altitude; $\lambda$ is the course angle.

Because of the various influencing factors of air target, especially the motion factors, we employed the short-cut route method to analyze the threat degree previously. However, this method cannot reach the correct and efficient result of threat degree. It ignores some kind of factors of the target movement which leads to some certain degree of distortion. The calculation method of the short-cut route $p$ can be obtained in Figure 2:

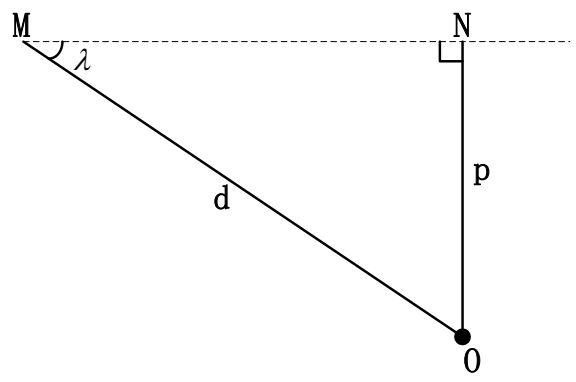

Figure 2. Short-cut route $\mathrm{p}$.

$O$ is the point of naval gun defense system; $M$ is the target point; $d$ is the slope distance; $\lambda$ is the course angle; $N$ is the point of the projection on the course. We can see the formula of the short-cut route $p$ in formula (1):

$p=d \sin (\lambda)$

\section{A NEW ANALYTICAL MODEL OF THREAT DEGREE}

\subsection{Build the relative function of threat degree}

\subsubsection{Function of slope distance}

We always consider the distance which is more than $10 \mathrm{~km}$ will have no threat to our defense system, so it should be 0 when it is more than $10 \mathrm{~km}$.

$\mu(d)=\left\{\begin{array}{cl}e^{-a d^{2}} & , d<10 \mathrm{~km} \\ 0 & , d<10 \mathrm{~km}\end{array}\right.$

$d$ is the slope distance, $\alpha$ is a constant.Take $\alpha=0.001 \mathrm{~km}^{-2}$ for the function.

\subsubsection{Function of flight speed}

The flight speed is the main reason which can influence the blast area of air defense. If the flight speed were different, it would have different threat degrees even if they are aiming to the same target. The threat degree range is as the same tendency as the range of flight speed.

$\mu(v)=1-e^{-a v}$

$v$ is the flight speed of target, $\alpha$ is a constant.

Take $\alpha=0.005 \mathrm{~s} / \mathrm{m}$ for the function. 
3.1.3 Function of flight altitude

If the target decreased its altitude, it would be more difficult to be found by the defense system. So it will be more aggressive to our defense system. Moreover, due to the previous experience, the target which has the altitude of more than $1 \mathrm{~km}$ will have none or only a small degree of threat to our defense system. We define the function of flight altitude in formula (4):

$\mu(h)=\left\{\begin{array}{cc}e^{-a h^{2}} & , h>1 k m \\ 1, & , h \leq 1 k m\end{array}\right.$

$h$ is the flight altitude of target, $\alpha$ is a constant,.Take $\alpha=0.005 \mathrm{~km}^{-2}$ for the function.

\subsubsection{Function of course angle}

When the course angle is greater than $90^{\circ}$, the target has no threat to our defense system because its course direction is opposite to our system. On the contrary, when the course angle is less than $90^{\circ}$, the threat degree is rapidly increased. So we define the function of course angle in formula (5):

$\mu(\lambda)=\left\{\begin{array}{c}e^{-\alpha \lambda}, \lambda \leq \frac{\pi}{2} \\ 0, \lambda>\frac{\pi}{2}\end{array}\right.$

$\lambda$ is the course angle, $\alpha$ is a constant. Take $\alpha=0.005$ for the function.

\subsection{Decision-making of threat degree}

For the threat degree of single target $\mathrm{X}_{\mathrm{i}}$, we use functions (1)-(5) to calculate the threat degree of each influencing factor. The threat degree of each factor can be obtained in formula (6):

$X_{i}=\left(\mu_{i}(d), \mu_{i}(v), \mu_{i}(h), \mu_{i}(\lambda)\right)$

If the target number is $n$, the $n \times 4$ decision-making matrix can be obtained in formula (7):

$$
\begin{aligned}
A & =\left(X_{0}, X_{1}, \ldots, X_{n-1}\right)^{T} \\
& =\left[\begin{array}{cccc}
x_{0}(1) & x_{0}(2) & x_{0}(3) & x_{0}(4) \\
x_{1}(1) & x_{1}(2) & x_{1}(3) & x_{1}(4) \\
\cdots & \ldots & \ldots & \ldots \\
x_{n-1}(1) & x_{n-1}(2) & x_{n-1}(3) & x_{n-1}(4)
\end{array}\right]
\end{aligned}
$$

Take the maximum value of each line in matrix $A$ for the best reference sequence $\alpha$, and take the minimum value of each line in matrix $A$ for the worst reference sequence $\beta$. They are in formula (8):

$\left\{\alpha=\left[\begin{array}{llll}x_{\max }(1) & x_{\max }(2) & x_{\max }(3) & x_{\max }(4)\end{array}\right]\right.$

$\left\{\beta=\left[\begin{array}{llll}x_{\min }(1) & x_{\min }(2) & x_{\min }(3) & x_{\min }(4)\end{array}\right]\right.$

Extend the best reference sequence $\alpha$ and the worst reference sequence $\beta$ to be matrixes which are the same in every line. After that, the difference ma- trixes of the best reference sequence $B$ and the difference matrixes of worst reference sequence $C$ can be obtained in formulas (9)-(10):

$B=\left[\begin{array}{cccc}x_{\max }(1) & x_{\max }(2) & x_{\max }(3) & x_{\max }(4) \\ x_{\max }(1) & x_{\max }(2) & x_{\max }(3) & x_{\max }(4) \\ \cdots & \cdots & \cdots & \cdots \\ x_{\max }(1) & x_{\max }(2) & x_{\max }(3) & x_{\max }(4)\end{array}\right]-A$

$C=A-\left[\begin{array}{cccc}x_{\min }(1) & x_{\min }(2) & x_{\operatorname{mix}}(3) & x_{\min }(4) \\ x_{\min }(1) & x_{\min }(2) & x_{\min }(3) & x_{\min }(4) \\ \ldots & \ldots & \ldots & \ldots \\ x_{\min }(1) & x_{\min }(2) & x_{\min }(3) & x_{\min }(4)\end{array}\right](10)$

Assume that the minimum value in matrix $\mathrm{B}$ is $x_{1}$ and the maximum value is $y_{1}$. The element of matrix $\mathrm{B}$ is $b_{i j}$. So the incidence coefficient matrix $D$ of the best reference sequence can be obtained in formula (11):

$d_{i j}=\frac{\left(x_{1}+\rho y_{1}\right)}{b_{i j}+\rho y_{1}}$

$\rho$ is the distinguishing coefficient, and the value range of it is $[0,1]$. Generally speaking, the differences between correlation coefficients can be improved if the value of $\rho$ decreased. We usually take $\rho=0.5$.

Similarly, the incidence coefficient matrix $E$ of the worst reference sequence can be also obtained by formula (11). The minimum value of matrix $C$ is $x_{1}$ and the maximum value is $y_{1}$. The element of matrix $C$ is $c_{i j}$.

Due to the different quality of each threat degree influencing element, the weight of each element in the matrix system is different. The total weight equals 1 . So we can define that if the weight of each influencing element is $W_{i}=\left[r_{1}, r_{2}, r_{3}, r_{4}\right]$, weights can be calculated by formula (12):

$w_{i}=\frac{r_{i}}{r_{1}+r_{2}+r_{3}+r_{4}}$

The best correlation threat degree $\eta$ from the best reference sequence and the worst correlation threat degree $\gamma$ from the worst reference sequence can be obtained in formula (13):

$\left\{\begin{array}{l}\eta=\sum_{k-1}^{4} w_{k} d_{o i}(k) \\ \gamma=\sum_{k-1}^{4} w_{k} e_{o i}(k)\end{array}\right.$

Finally, we use the best correlation threat degree $\eta$ and worst correlation threat degree $\gamma$ to calculate the comprehensive correlation threat degree of each target $\delta$ in formula (14):

$\delta=\frac{1}{\left(1+\frac{\gamma}{\eta}\right)^{2}}$

If the value of the comprehensive correlation threat degree becomes greater, our defense system rates will become greater for the threat degree. It is the finally 
determinative element of air assault threat analysis.

\section{ANALYSIS OF APPLICATION EXAMPLE AND COMPARATION OF RESULTS}

The practical example of 8 targets is shown in Table 1, and the elements of influencing factors of them are $X=\left(X_{1}, X_{2}, \ldots, X_{8}\right)^{T}$.

Table 1. Influencing factors of multi-targets

\begin{tabular}{lllll}
\hline Setting & $\begin{array}{l}\text { Slope } \\
\text { distance }\end{array}$ & Flight speed & $\begin{array}{l}\text { Flight } \\
\text { altitude }\end{array}$ & $\begin{array}{l}\text { Course } \\
\text { angle }\end{array}$ \\
\cline { 2 - 5 } & $\mathrm{km}$ & $\mathrm{m} / \mathrm{s}$ & $\mathrm{km}$ & $\circ$ \\
\hline Target 1 & 12 & 200 & 8 & 20 \\
Target 2 & 55 & 500 & 3.5 & 40 \\
Target 3 & 40 & 120 & 0.5 & 15 \\
Target 4 & 25 & 250 & 8 & 30 \\
Target 5 & 30 & 300 & 7 & 100 \\
Target 6 & 28 & 400 & 2 & 52 \\
Target 7 & 36 & 450 & 1.4 & 15 \\
Target 8 & 20 & 150 & 10 & 5 \\
\hline
\end{tabular}

\subsection{Threat decision-making of the short-cut route}

First, we use the traditional method of short-cut route to analyze the threat degree of the 8 practical examples.

Previously, we introduce the evaluation method of this method. According to the value of course angle, we can use formula (1) to calculate the threat degree decision-making matrix $P$ of the multi-targets.

$P=[4.1042,35.353,10.353,12.5,29.544,22.064$, $9.3175,1.7431]$

The threat degree of short-cut route is shown by Figure 3:

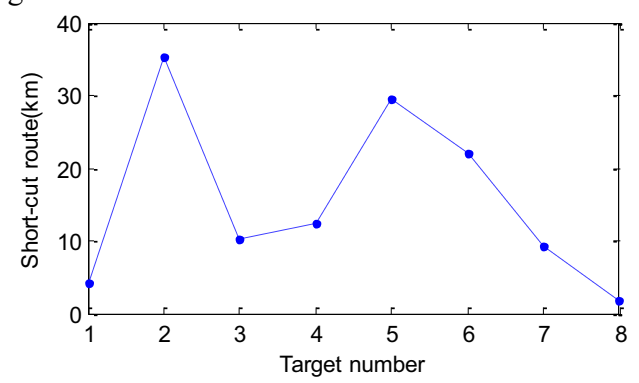

Figure 3. Threat degree analysis of short-cut route.

Therefore, through Figure 3, we can easily found that the threat rank of the 8 targets.

$$
\mathrm{x}_{2}>\mathrm{x}_{5}>\mathrm{x}_{6}>\mathrm{x}_{4}>\mathrm{x}_{3}>\mathrm{x}_{7}>\mathrm{x}_{1}>\mathrm{x}_{8}
$$

We can see that the greatest one is target 2 , and the lowest one is target 8 .

\subsection{Threat decision-making of the grey correlation}

Second, we use the new model to analyze the threat degree of the 8 example targets. The new method analyzes the threat influencing element of slope distance, flight speed, flight altitude and the course angle. With the definition of functions relate to them, we can calculate the threat contribution of each factor. In the end, use the reference sequence and relation coefficient to determine the final threat degree of each target.

According to the weight decision functions (2)-(5), we can obtain the $8 \times 4$ dimension decision-making matrix A.

$A=\left[\begin{array}{llll}0.86589 & 0.63212 & 0.72615 & 0.90484 \\ 0.04856 & 0.91792 & 0.94059 & 0.81873 \\ 0.20190 & 0.45119 & 1.00000 & 0.92774 \\ 0.53526 & 0.71350 & 0.72615 & 0.86071 \\ 0.40657 & 0.77687 & 0.78271 & 0.00000 \\ 0.45658 & 0.86467 & 0.98020 & 0.77105 \\ 0.27362 & 0.89460 & 0.99025 & 0.92774 \\ 0.67032 & 0.52763 & 0.60653 & 0.97531\end{array}\right]$

Matrix $A$ provides the best reference sequence which is $[0.86589,0.91792,1.0,0.97531]$, and the worst reference sequence which is $[0.04856,0.45119,0.60653,0.0]$.

By using formula (9), we can obtain the difference matrixes of the best reference sequence $B$.

$B=\left[\begin{array}{llll}0.00000 & 0.28580 & 0.27385 & 0.07047 \\ 0.81733 & 0.00000 & 0.05941 & 0.15658 \\ 0.66399 & 0.46673 & 0.00000 & 0.04757 \\ 0.33063 & 0.20442 & 0.27385 & 0.11460 \\ 0.45932 & 0.14105 & 0.21729 & 0.97531 \\ 0.40931 & 0.05325 & 0.01980 & 0.20426 \\ 0.59227 & 0.02332 & 0.00975 & 0.04757 \\ 0.19557 & 0.39029 & 0.39347 & 0.00000\end{array}\right]$

The minimum value of matrix $\mathrm{B}$ is $x_{1}=0.00000$ and the maximum value is $y_{1}=0.97531$. According to formula (11), we can obtain the incidence coefficient matrix of the best reference sequence $D$.

$D=\left[\begin{array}{llll}1.00000 & 0.63049 & 0.64038 & 0.87374 \\ 0.37369 & 1.00000 & 0.89140 & 0.75695 \\ 0.42344 & 0.51096 & 1.00000 & 0.91112 \\ 0.59595 & 0.70463 & 0.64038 & 0.80972 \\ 0.51496 & 0.77565 & 0.69176 & 0.33333 \\ 0.54367 & 0.90155 & 0.96098 & 0.70479 \\ 0.45156 & 0.95436 & 0.98040 & 0.91112 \\ 0.71375 & 0.55545 & 0.55345 & 1.00000\end{array}\right]$

According to formula (12), we can obtain the weight of each influencing element $W_{i}$. They are shown in Table 2:

Table 2. Weight of each influencing element in the best reference sequence.

\begin{tabular}{llccl}
\hline Setting & $\begin{array}{l}\text { Slope } \\
\text { distance }\end{array}$ & Flight speed & $\begin{array}{l}\text { Flight } \\
\text { altitude }\end{array}$ & $\begin{array}{l}\text { Course } \\
\text { angle }\end{array}$ \\
\hline Target 1 & 0.31800 & 0.20050 & 0.20364 & 0.27786 \\
Target 2 & 0.12365 & 0.33090 & 0.29497 & 0.25048 \\
Target 3 & 0.14881 & 0.17957 & 0.35143 & 0.32019 \\
\hline
\end{tabular}




\begin{tabular}{lllll}
\hline Target 4 & 0.21666 & 0.25616 & 0.23281 & 0.29437 \\
Target 5 & 0.22238 & 0.33494 & 0.29873 & 0.14395 \\
Target 6 & 0.17476 & 0.28979 & 0.30890 & 0.22655 \\
Target 7 & 0.13694 & 0.28943 & 0.29732 & 0.27631 \\
Target 8 & 0.25287 & 0.19678 & 0.19607 & 0.35428 \\
\hline
\end{tabular}

Use the data calculated before in Table 2, we can obtain the best correlation threat degree $\eta$ of those 8 targets by formula (13).

$\eta=[0.81760,0.82964,0.79793,0.69706,0.62895$,

$0.81279,0.88130,0.75258]$

Similarly, by using the worst reference sequence $[0.04856,0.45119,0.60653,0.0]$, we can obtain the difference matrixes of the worst reference sequence $C$ by formula (10).

$C=\left[\begin{array}{llll}0.81733 & 0.18093 & 0.11962 & 0.90484 \\ 0.00000 & 0.46673 & 0.33406 & 0.81873 \\ 0.15334 & 0.00000 & 0.39347 & 0.92774 \\ 0.48670 & 0.26231 & 0.11962 & 0.86071 \\ 0.35801 & 0.32568 & 0.17618 & 0.00000 \\ 0.40802 & 0.41348 & 0.52901 & 0.77105 \\ 0.22506 & 0.44341 & 0.38372 & 0.92774 \\ 0.62176 & 0.07644 & 0.00000 & 0.97531\end{array}\right]$

The minimum value of matrix $C$ is $x_{1}=0.00000$ and the maximum value is $y_{1}=0.97531$. The incidence coefficient matrix of the worst reference sequence $E$ can be calculated by formula (11).

$E=\left[\begin{array}{llll}0.37369 & 0.72938 & 0.80302 & 0.35020 \\ 1.00000 & 0.51096 & 0.59346 & 0.37329 \\ 0.76078 & 1.00000 & 0.55345 & 0.34454 \\ 0.50049 & 0.65024 & 0.80302 & 0.36166 \\ 0.57665 & 0.59957 & 0.73460 & 1.00000 \\ 0.54446 & 0.54116 & 0.47966 & 0.38743 \\ 0.68422 & 0.52376 & 0.55964 & 0.34454 \\ 0.43956 & 0.86449 & 1.00000 & 0.33333\end{array}\right]$

The weight of each influencing element $W_{i}$ is shown in Table 3:

Table 3. he weight of each influencing element in the worst reference sequence.

\begin{tabular}{llccl}
\hline Setting & $\begin{array}{l}\text { Slope } \\
\text { distance }\end{array}$ & Flight speed & $\begin{array}{l}\text { Flight } \\
\text { altitude }\end{array}$ & $\begin{array}{l}\text { Course } \\
\text { angle }\end{array}$ \\
\hline Target 1 & 0.16563 & 0.32328 & 0.35591 & 0.11522 \\
Target 2 & 0.40360 & 0.20622 & 0.23952 & 0.15066 \\
Target 3 & 0.28614 & 0.37611 & 0.20816 & 0.12959 \\
Target 4 & 0.21616 & 0.28083 & 0.34682 & 0.15620 \\
Target 5 & 0.19810 & 0.20598 & 0.25237 & 0.34355 \\
Target 6 & 0.27882 & 0.27713 & 0.24564 & 0.19841 \\
Target 7 & 0.32395 & 0.24797 & 0.26496 & 0.16312 \\
Target 8 & 0.16667 & 0.32778 & 0.37916 & 0.12639 \\
\hline
\end{tabular}

From the data which is shown in Table 3 and formula (13), we can obtain the worst correlation threat degree $\gamma$ of those 8 targets.

$\gamma=[0.62384,0.70736,0.75365,0.62579,0.76667$, $0.49647,0.55601,0.77791]$

Put $\eta$ and $\gamma$ into formula (14), we can finally obtain the comprehensive correlation threat degree of each target $\delta$. $\delta=[0.56721,0.53978,0.51427,0.52694,0.45066$, $0.62080,0.61316,0.49172]$

The figure which demonstrated the comprehensive correlation threat degree of each target is shown in Figure 4:

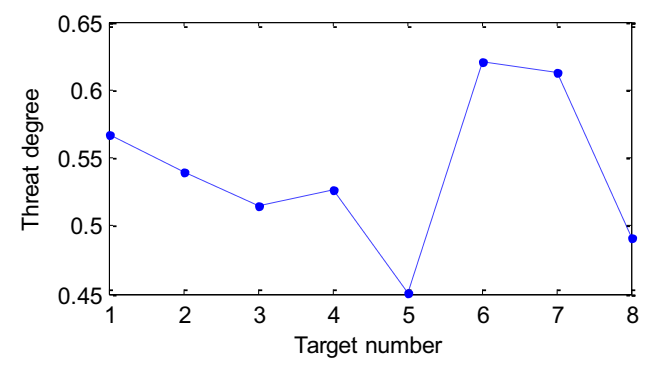

Figure 4. Curve graph of comprehensive correlation threat degree.

As it is shown in Figure 4, we can obtain the final threat rank of each target.

$$
x_{6}>x_{7}>x_{1}>x_{2}>x_{4}>x_{3}>x_{8}>x_{5}
$$

We can see the target 6 is the greatest one in term of threat degree while the lowest one is target 5 .

According to the discussion from the specialists and the practical applications, the threat rank which is calculated from the new model is an efficient and accurate method for the defense system. In addition, our method can be easily used in modern warfare.

\subsection{Contrastive analysis of threat decision-making}

From the previous analysis of threat decision-making, we obtained two different threat ranks of 8 targets.

4.3.1 Result analysis of the short-cut route method From 2.1, we obtained the threat rank from short-cut route method:

$$
x_{2}>x_{5}>x_{6}>x_{4}>x_{3}>x_{7}>x_{1}>x_{8}
$$

Take target 2 and target 5 as the examples. As for target 2 , although it reaches the greatest value according to the short-cut route, it ignores two main factors (flight speed and flight altitude) because of the fundamental reason of the method.

As for target 5 , its course angle is more than $90^{\circ}$ and its flight direction is opposite to our defense system, so it has no such practical threat.

As for target 1, the decision-making of target 2 and target 5 obviously have a certain degree of distortion. Although this method can be used in some certain circumstance, it cannot receive the correct threat rank of every target due to the ignorance of other influencing factors.

\subsubsection{Result analysis of the new model method}

From 2.2, we obtain the threat rank from the method of new analysis model: 


$$
\mathrm{x}_{6}>\mathrm{x}_{7}>\mathrm{x}_{1}>\mathrm{x}_{2}>\mathrm{x}_{4}>\mathrm{x}_{3}>\mathrm{x}_{8}>\mathrm{x}_{5}
$$

Also, we take the same target (target 6 and target 5) as examples to compare with the performance of two methods.

As for target 6, although its short-cut route is not the minimum one, its threat degree is greater because of the higher speed and the lower flight altitude. It takes the flight altitude and flight speed into consideration which are very essential for the threat degree analysis. From our experience, we know that the low altitude can lead the distortion of detection radar which can be more aggressive than the one with higher altitude. In addition, the high speed target can lead to the lower defense efficiency and influence the lethal zone of naval gun. Therefore, they are also the essential factors for our threat degree assessment. We can also see that in the method of short-cut route, and the threat degree of target 6 is greater (it achieves the third place in rank).

As for target 5, it has no such threat to our defense system due to its course angle which is more than $90^{\circ}$. So the threat degree of target 5 should acquire the lowest value. However, in the method of short-cut route, it is secondary in terms of threat degree. Therefore, it is obviously that the previous method has a certain degree of distortion.

According to the analysis results, we can see that the method of short-cut route has a certain degree of distortion and cannot reflect the accurate threat degree of the targets. However, the new analytical model, which takes every relevant influencing factor into account, is more considerable and more accurate for the decision-making in the defense system.

As for the consideration of computing time, we use practical application to verify the usability of the new method. The final consumption of time which is used by the new method is in the range of the theoretical time. As for the consideration of the consumption of system recourse, new system has no such complex calculation and it can be used in any condition of circumstances.

\section{CONCLUSION}

For the modern warfare, it has become stricter that the naval gun defense system must give the correct action immediately. Because of the multi-targets threat, the threat degree analysis has become the essential thing to overcome in modern society. Correct decision and efficient defense can prevent the defense system from destroying and even can make a great change for the whole war situation.

This paper uses a new analytical model of grey correlation with practical examples to analyze the threa degree, and uses the result which is calculated from it to compare with the result calculated from the old method of short-cut route. From the contrastive analysis, we can find that the new model is efficient and accurate that behaves very well in practical application, while the old method always cannot give out the correct threat degree rank and has a certain degree of distortion. This new model can be used in theoretical time range and it has very little consumption of the system recourse.

As we all know, for example, the bombardment aircraft has a greater level of threat degree than reconnaissance plane. However, due to the diversity of target character, the threat level of different kinds of targets cannot be measured. This problem should be possibly solved in the future.

\section{ACKNOWLEDGEMENT}

The authors appreciate the computing assistance which is offered by Ph.D. candidates Shuai Zhao and Professor Yuan Zhang from the University of Northwestern Polytechnical University. The authors also acknowledge the helpful comments and suggestions from the editor.

\section{REFERENCES}

[1] Das, Sumanta Kumar. 2014. Modeling intelligent decision-making command and control agents: An application to air defense. Intelligent Systems, IEEE, 29(5): 22-29.

[2] Philip R. E. Cutler and X. Thong Nguyen. 2003. Description of a rule-based model for the automatic allocation of airborne assets. $6^{\text {th }}$ International Conference on Information Fusion.

[3] LU Hua, ZHOU Deyun, ZHANG Kun. 2009. Research on unascertained-measure-based tactics decision of air-to-ground multi-target Combat. 2009 IEEE International Conference on Intelligent Computing and Intelligent Systems.

[4] Jeong Hoon Kim, Chang Beom Choi, Tag Gon Kim. 2011. Battle experiments of naval air defense with discrete event system-based mission-level modeling and simulations. The Journal of Defense Modeling \& Simulation, 8(3)

[5] Jun Wang, Xiaozhe Zhao, Yinhan Zhang, Buyun Wang. 2011. Cooperative air-defense system of system immune multi-agent model based on danger theory for surface warship formation. International Conference on Computational and Information Sciences, ICCIS 2011.

[6] Jun Wang, Xiaozhe Zhao, Beiping Xu, Wei Wang, Zhiyong Niu. 2013. Immune multi-agent model using vaccine for cooperative air-defense system of systems for surface warship formation based on danger theory. Journal of Systems Engineering and Electronics, (6): 946-953

[7] Hangyu Wang, Jieshi Wang, Peng Li. 2006. Shipborne Fire Control Theory. Beijing: National Defense Industry Press.

[8] D.F. Li. 2010. TOPSIS-based nonlinear-programming methodology for multi-attribute decision making with 
interval-valued intuitionistic fuzzy sets. IEEE Trans. On Fuzzy Systems, 18(2): 299-311.

[9] Yongjie Xu, Yongchun Wang, Xudong Miu. 2012. Multi-attribute decision making method for air target threat evaluation based on intuitionistic fuzzy sets. Journal of Systems Engineering and Electronics, 23(6): 891-897.

[10] J. Ye. 2010. Fuzzy decision-making method based on the weighted correlation coefficient under intuitionistic fuzzy environment. European Journal of Operational Research, 205(2): 202-204.

[11]Liebhaber M J, Smith C A P. 2000. Naval air threat assessment: Cognitive factors and model. $5^{\text {th }}$ International Command and Control Research and Technology Symposium.

[12] Lu Huiying, Peng Wu, Wang Ruimei. 2014. A real-time network threat recognition and assessment method based on association analysis of time and space. Journal of Computer Research and Development, 51(5): 1039-1049.

[13]Yuan Haiying, Gao Xuejin. 2010. The weapon target assignment strategy research on genetic algorithm. 2010

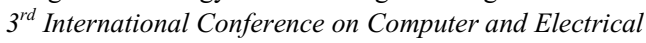
Engineering.

[14] Sifeng Liu. 2010. Theory and Application of Grey System. NSW: Science Press.

[15]Yin Zhihong, Cui Naigang, Guan Shiyi. 2007. A threat coefficient method for extended air defense system analysis. Second International Conference on Space Information Technology. 